International Mathematical Forum, 1, 2006, no. 11, 517-521

\title{
SEQUENCES FROM INTEGER TETRAHEDRONS
}

\author{
T. Aaron Gulliver \\ Department of Electrical and Computer Engineering \\ University of Victoria, P.O. Box 3055, STN CSC \\ Victoria, BC, Canada V8W 3P6 \\ agullive@ece.uvic.ca
}

\begin{abstract}
This paper presents a number of sequences based on integers arranged in a three-dimensional array which is a tetrahedron. This approach provides a simple derivation of some well known sequences. In addition, a number of new integer sequences are obtained.
\end{abstract}

Keywords: integer arrays, integer sequences

\section{Mathematics Subject Classification: $11 Y 55$}

\section{Introduction}

In a previous paper [1], several well-known sequences (and many new sequences), were derived from two-dimensional arrays of integers. In this paper, we consider the case of a three-dimensional array which is a three-sided pyramid or tetrahedron of integers. For example, the number of elements in the tetrahedron is

$$
s_{n}=1+3+6+10+15+\ldots+n(n+1) / 2=\sum_{i=1}^{n} i(i+1) / 2=\frac{1}{6} n(n+1)(n+2),
$$

where $n$ is the number of levels. Starting from $n=1$, we have

$$
1,4,10,20,35, \ldots
$$

which is sequence A000292 in the On-Line Encyclopedia of Integer Sequences [2], and is appropriately called the tetrahedral or pyramidal numbers. Additional sequences based on this integer array structure are given in the next section. 


\section{Tetrahedral Pyramids of Integers}

A tetrahedral array of integers has the following structure for $n=1$ to 4

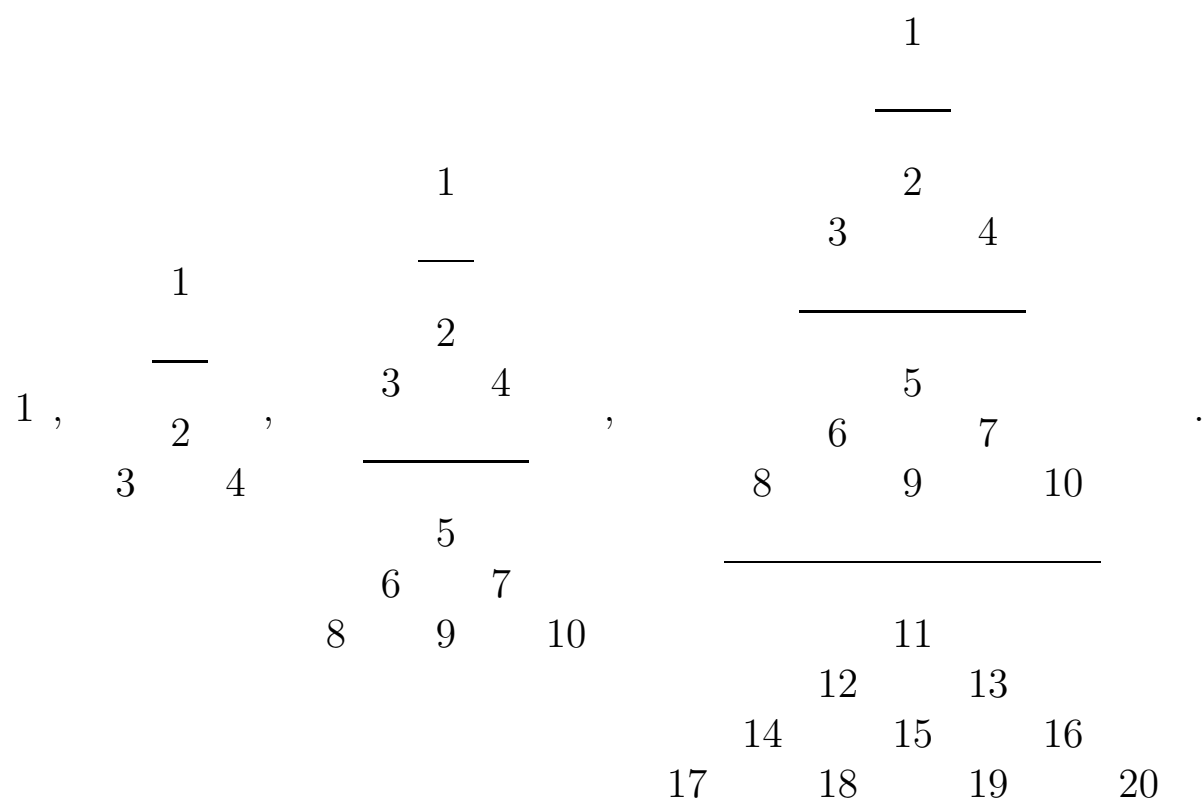

In addition to (1), the following sequences are obtained from the corners of the pyramids

$$
\begin{array}{llllll}
1, & 2, & 5, & 11, & 21, & \ldots \\
1, & 3, & 8, & 17, & 31, & \ldots
\end{array} .
$$

The first of these is sequence A050407, and is given by

$$
s_{n}=\frac{1}{6}(n+2)\left(n^{2}-2 n+3\right)
$$

which is just one more than the tetrahedral numbers. The second is sequence A105163 (with the leading 1 removed), given by

$$
s_{n}=\frac{1}{6}\left(n^{3}+3 n^{2}-4 n+6\right) \text {. }
$$

In this case, the sequence $s_{n}-1$ is the $x^{3}$ term in $\left(1+x+x^{2}\right)^{n}$ (A005581 in the Encyclopedia). The sum of the elements along the edges give three sequences

$1, \quad 3, \quad 8, \quad 19, \quad 40, \quad \ldots$

$1,4,12,29,60, \ldots$,

$1, \quad 5, \quad 15, \quad 35, \quad 70, \ldots$ 
with

$$
\begin{aligned}
& s_{n}=\frac{1}{24} n\left(n^{3}+2 n^{2}-n+22\right) \\
& s_{n}=\frac{1}{24} n\left(n^{3}+6 n^{2}-n+18\right), \\
& s_{n}=\frac{1}{24} n(n+1)(n+2)(n+3)
\end{aligned}
$$

respectively. The third sequence is A000332, and is easily recognized as the binomial coefficient $\left(\begin{array}{c}n \\ 4\end{array}\right)$. The second is A086274, while the first sequence is new. Unless indicated otherwise, all subsequent sequences are new. Taking the sum of the three sequences in (2) gives

$$
3, \quad 12, \quad 35,83,170, \ldots,
$$

with

$$
s_{n}=\frac{1}{24} n\left(3 n^{3}+14 n^{2}+9 n+46\right) .
$$

The sum of the elements on the edges is then (3) -2

$$
1, \quad 10,33,81,168, \ldots,
$$

with

$$
s_{n}=\frac{1}{24}\left(3 n^{4}+14 n^{3}+9 n^{2}+46 n-48\right) .
$$

The sum of the rows on the faces of the pyramid give the sequences

$$
\begin{array}{llllll}
1, & 5, & 19, & 54, & 125, & \ldots \\
1, & 6, & 22, & 60, & 135, & \ldots \\
1, & 7, & 27, & 74, & 165, & \ldots
\end{array}
$$

with

$$
\begin{aligned}
& s_{n}=\frac{1}{6} n\left(n^{3}+n^{2}-n+5\right) \\
& s_{n}=\frac{1}{6} n(n+1)\left(n^{2}+2\right), \\
& s_{n}=\frac{1}{6} n\left(n^{3}+3 n^{2}-n+3\right)
\end{aligned}
$$

respectively. From (5), we can obtain the sum of all elements on each face which are the sequences

$1, \quad 6, \quad 25, \quad 79, \quad 204, \ldots$

$1, \quad 7, \quad 29, \quad 89, \quad 224, \ldots$,

$1, \quad 8, \quad 35, \quad 109, \quad 274, \ldots$ 
with

$$
\begin{aligned}
& s_{n}=\frac{1}{120} n(n+1)\left(4 n^{3}+11 n^{2}-n+4\right) \\
& s_{n}=\frac{1}{120} n(n+1)(n+2)\left(4 n^{2}+3 n+13\right), \\
& s_{n}=\frac{1}{120} n(n+1)\left(4 n^{3}+21 n^{2}+9 n+26\right)
\end{aligned}
$$

respectively. Summing the sequences in (6) gives the sequence for the sum of the elements on all three faces (if the apex is counted once for each face)

$$
3, \quad 21, \quad 89, \quad 277, \quad 702, \ldots,
$$

with the terms given by the formula

$$
s_{n}=\frac{1}{120} n(n+1)\left(12 n^{3}+43 n^{2}+27 n+98\right) .
$$

Adding the elements on a level gives

$$
1, \quad 9, \quad 45, \quad 155, \quad 420, \ldots
$$

with

$$
s_{n}=\frac{1}{24} n(n+1)(n+2)\left(2 n^{2}-n+3\right) .
$$

This is sequence A095166, the natural numbers arranged in triangles. Note that the formula in [2] differs from that above because the sequence here begins at $n=1$, as opposed to $n=0$. The sum of all elements is the sum for 1 to $n$ levels which is

$$
1, \quad 10, \quad 55, \quad 210,630, \ldots
$$

with terms

$$
s_{n}=\frac{1}{72} n(n+1)(n+2)(n+3)\left(n^{2}+2\right) .
$$

If one takes the sum of the faces (7) and subtracts the sum of the edges (3), we obtain

$$
0, \quad 9, \quad 54, \quad 194, \quad 532, \ldots
$$

with

$$
s_{n}=\frac{1}{30} n(n-1)\left(3 n^{3}+13 n^{2}+13 n+33\right) .
$$

Adding one to this total to include the apex (as it has been added three times and subtracted three times), gives

$$
1, \quad 10, \quad 55, \quad 195, \quad 533, \ldots
$$

which is the sum of the elements on the surface of the tetrahedron. The corresponding sequence terms are given by the formula

$$
s_{n}=\frac{1}{30}\left(3 n^{5}+10 n^{4}+20 n^{2}-33 n+30\right) .
$$


The sum of the edge elements on each level of the pyramid gives the sequence

$$
3, \quad 9, \quad 23, \quad 48,87, \ldots
$$

with

$$
s_{n}=\frac{1}{2}\left(n^{3}+2 n^{2}-n+4\right)
$$

Note that the apex is counted three times as it is part of each edge. The sum of the face elements on each level is the sequence

$$
3, \quad 18, \quad 68, \quad 188, \quad 425, \ldots
$$

with

$$
s_{n}=\frac{1}{6} n\left(3 n^{3}+5 n^{2}+10\right)
$$

where again the apex is counted three times. Taking the difference (14)-(13) gives the sequence

$$
0, \quad 9, \quad 45, \quad 140,338, \ldots
$$

with

$$
s_{n}=\frac{1}{6}(n-1)\left(3 n^{3}+5 n^{2}-n+12\right)
$$

Summing these elements starting from level 1 and adding 1 for the apex gives (11), as expected.

\section{References}

[1] T.A. Gulliver, Sequences from Arrays of Integers, Int. Math. Journal 1 323-332 (2002).

[2] N.J.A. Sloane, On-Line Encyclopedia of Integer Sequences, http://www.research.att.com/ ^ njas/sequences/index.html.

Received: May 12, 2005 